\title{
Ce que vous devez savoir du PDCA (Plan, do, check, act)
}

\author{
Rafaël Perez Uribe
}

\section{Le cycle PDCA}

Tant aux niveaux entrepreneurial qu'académique, un des aspects qui a le plus attiré mon attention dans l'utilisation du cycle PDCA (PFVA) c'est sa similitude avec l'exercice de prise de décisions que les êtres humains effectuent à chaque instant.

En pensant à ce que nous allons faire, nous analysons des réalités, nous tamisons des données et nous les transformons en information afin de choisir ce qui nous sert. L'action sélectionnée est exécutée en la confrontant à ce que nous avions pensé initialement pour ne conserver que les phases utiles. Par contre, nous ne répétons pas les actes sans résultats ou qui ont entraîné de mauvais résultats. De façon permanente ce cycle est répété de façon automatique comme si nous respirions ou marchions; c'est tout simplement un acte réflexe.

$\mathrm{Au}$ siècle passé, durant les années 30 , le Docteur W. A. Shewhart ${ }^{1}$ du Bell Laboratories, a brillamment développé les bases du contrôle de qualité moderne et du contrôle de qualité statistique des processus (charte de Shewhart). Il a aussi commencé à appliquer le cycle PDCA au secteur manufacturier.

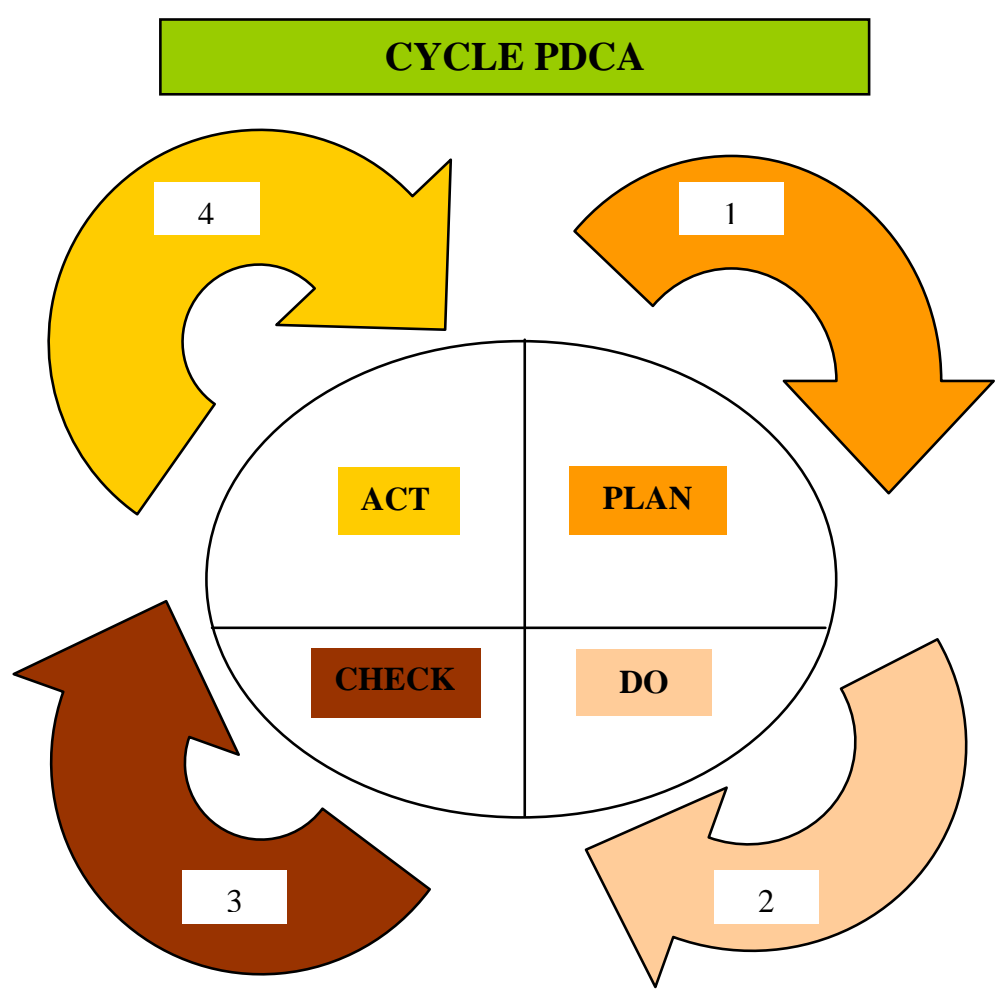


Pour le secteur manufacturier, les étapes de base du PDCA (PFVA) peuvent se résumer ainsi :

\section{Planifier :}

- Impliquer des personnes clés pour structurer un plan d'action.

- Compiler les données disponibles.

- Comprendre les besoins des clients, la situation qui est à l'origine d'un problème ou encore qui rend vulnérable un processus donné.

- Étudier de façon exhaustive le/les processus impliqués. Un processus est-il capable d'accomplir ce qui est nécessaire?

- Structurer le plan.

- Former le personnel et créer un scénario adéquat pour que les employés soient tenus de développer les activités proposées dans le plan afin d'atteindre les objectifs établis.

\section{Faire :}

- Exécuter les activités proposées en fonction des ressources assignées ou mettre en œuvre l'amélioration en vérifiant les causes des problèmes.

- Compiler les données appropriées.

- Superviser les temps d'exécution et les ressources.

\section{Vérifier :}

- Le dévoilement et l'analyse des données.

- Que les résultats escomptés sont atteints.

- Les différences avec le plan initial: les comprendre et les documenter.

- Les problèmes et les erreurs.

- Qu'est-ce qui a été appris ?

- Que reste-t-il à résoudre ?

\section{Agir :}

- Réflexion sur ce qui a été appris durant les phases planifier, faire et vérifier.

- Actions correctives à effectuer.

- Dans le cas d'un projet d'amélioration, présenter les résultats à la direction pour évaluation.

- Standardisation des activités, des outils, des indicateurs et des améliorations qui ont eu des résultats excellents dans le cycle.

- Incorporer l'amélioration au processus en question.

- Communiquer l'amélioration à tous les membres de l'entreprise.

- Identifier de nouveaux projets/problèmes.

\section{Répéter le processus autant de fois que nécessaire afin que l'organisation atteigne un état stable de qualité ${ }^{2}$.}

À partir de l'année 1950 et de façon répétée pendant les deux décennies suivantes, le professeur Deming ${ }^{3}$ a présenté le Cycle PDCA dans l'introduction de chacune des formations offertes à la haute direction des entreprises japonaises.

Depuis lors, le cycle PDCA (PFVA) est devenu le symbole incontesté de l'Amélioration Continue au niveau mondial ${ }^{4}$.

De fait, les prix nationaux et internationaux de qualité sont basés sur le cycle PDCA et le système de gestion de la qualité des Normes ISO 9000 : 2000, s'appuie sur le schème d'Amélioration Continue de Qualité de ce Cycle.

Comme vu précédemment, les étapes du Cycle PDCA requièrent la compilation et l'analyse d'une quantité substantielle de données. Pour accomplir l'objectif d'amélioration continue des mesures 
objectives nécessaires doivent être effectuées et un consensus obtenu tant dans la définition des objectifs/problèmes que dans l'utilisation d'indicateurs appropriés. Le cycle peut être complémenté par une série d'outils fondés sur des techniques statistiques simples. Pour n'en mentionner que quelques unes : Diagramme d'Ishikawa ou de Cause-Effets, Liste de Vérification (check list), Diagramme de Flux, Distribution de Fréquence et Histogramme, Diagramme de Pareto, Charte de Contrôle.

Le PDCA est utilisé dans le modèle KAIZEN (amélioration continue à petits pas) qui est à l'origine du miracle économique japonais des années 70 et qui fut popularisé par Masaaki Imai ${ }^{5}$.

Il est également employé dans la version américaine du Contrôle Total de la Qualité (CTQ) ${ }^{6}$ d'Armand Feigenbaum ${ }^{7}$.

A la fin des années 70 et durant les années 80, ceux qui, comme nous, eurent l'audace d'utiliser les cercles de contrôle de qualité en se servant du cycle de Deming comme outil fondamental de base obtinrent des améliorations surprenantes. D'autre part, bien que le modèle de standardisation proposé par la JUSE $^{8}$ recommandait dès 1971 une documentation précise des pratiques de gestion, en occident cette standardisation ne prend force qu'avec l'apparition de la norme ISO $9000^{9}$ en 1987.

Ishikawa $^{10}$, le père des cercles de qualité au Japon, a amplifié les quatre étapes du cycle de Deming en y ajoutant les six points suivants :

- Définir des objectifs.

- Définir les méthodes pour atteindre ces objectifs.

- Éduquer et entraîner le personnel.

- Mettre en œuvre les activités prévues au plan.

- Vérifier les effets de la mise en œuvre.

- Prendre les actions appropriées.

Ces six points se distribuent dans la roue PDCA de la manière suivante :

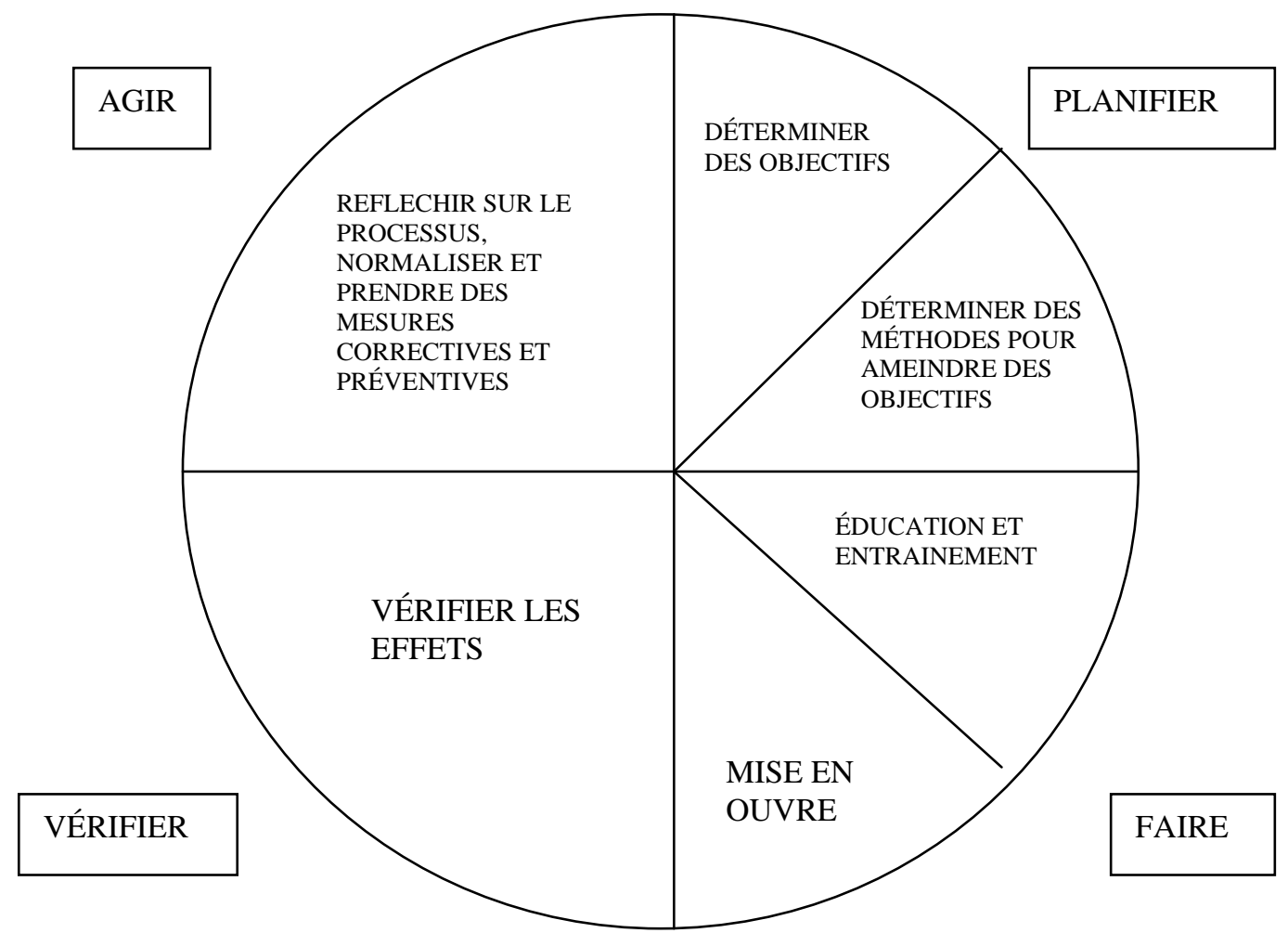


Selon le «Grupo Kaizen SA» ${ }^{11}$ «le processus d'amélioration de la qualité requiert plusieurs retours sur le cycle, qui peuvent être schématisés comme un ensemble de cercles remontant une pente. L'absence de données sur l'amélioration, ainsi que d'un système de gestion, introduit de l'entropie qui provoque le recul du cercle à un état antérieur» Quesada M., qui fait partie du groupe, affirme que le PDCA peut être utilisé avec la norme ISO 9000 qui, combinée avec le modèle d'Ishikawa, peut se formuler ainsi :

\section{Planifier}

1. Identifier des produits

2. Identifier des clients

3. Identifier les demandes des clients (5.2)

4. Définir les spécifications des demandes du client (7.1)

5. Identifier les étapes clef du processus (diagramme de flux) (7.5)

6. Identifier et choisir les paramètres de mesure (8.1)

7. Déterminer la performance du processus $(8.2 .3,8.2 .4)$

8. Identifier des normes de comparaison (benchmarks) (5.1 d'ISO 9004)

Faire

9. Identifier les opportunités d'amélioration (8.5)

10. Développer un plan pilote

11. Mettre en œuvre les améliorations

Vérifier

12. Évaluer l'efficacité (8.2, 8.5.2)

Agir

13. Institutionnaliser l'amélioration et/ou passer à l'étape 9 (5.6)
Selon Ramirez ${ }^{12}$ (2005), ce cycle peut être également utilisé dans un processus de négociation de la manière suivante :

\section{Planifier}

- Objectiver la négociation en préparant tous ses éléments.

- Définir ses enjeux et séparer les enjeux de chaque position.

- Quel est l'objectif de cette négociation ? Quel élément est prêt à céder l'autre partie?

- Quelles informations supplémentaires sont nécessaires pour atteindre mes objectifs?

- Intérêts en cours

- Alternatives possibles

- Visées possible

- Aspects humains, personnalités, niveau de tolérance, etc.

- Comment devrait se dérouler la négociation?

- Quelle information additionnelle ai-je besoin lors de la négociation?

- Quel type de questions ouvertes doisje poser afin d'obtenir le plus d'informations utiles ?

C'est une étape clef de la négociation, mais celle-ci reste sans résultat si la suivante n'est pas effectuée :

\section{Faire}

- Exécuter ce qui est projeté. Pour chaque action projetée, il doit exister un pourquoi, afin de réaliser les objectifs des enjeux définis préalablement. En ne suivant pas un schéma prédéterminé la possibilité de faire des erreurs est élevée. Toutefois, ceci ne signifie pas que tout ce qui est 
prévu ne puisse pas être changé dans les détails. A ce stade ci, on entre dans l'étape suivante du cycle :

\section{Vérifier}

- Contrôler l'atteinte ou non de l'objectif général prévu initialement. Il est possible que les actions prévues ne donnent pas le résultat attendu. C'est pourquoi il est important dans une négociation d'identifier des objectifs spécifiques qui permettent l'atteinte finale de l'objectif général. En réévaluant l'exercice ou les variables critiques de la négociation, une remise en question est possible pour générer de nouvelles options. Si on ne possède pas une ouverture d'esprit suffisante pour produire de nouvelles idées on risque fort de passer à un conflit des positions compliquant ainsi la négociation.

\section{Conclusion}

Comme vu précédemment, depuis plus de soixante ans le cycle PDCA a prouvé son immense utilité comme outil de gestion. Il est pertinent, efficace, efficient et peut s'appliquer à beaucoup de sujets et de
Agir

- Prendre des décisions justes et rapides mais seulement si la quantité d'information recueillie durant les étapes de Planifier et Agir est jugée suffisante. Ce sont fondamentalement deux types de décisions : continuer avec le plan si ce qui était prévu est obtenu ou bien redéfinir des actions avec comme base les objectifs spécifiques proposés initialement. Surtout ne pas changer d'objectif général, car cela créerait un autre problème ou une autre ronde de négociation, revoir seulement les actions prévues afin d'identifier celles permettant d'atteindre cet objectif.

\section{Bibliographie}

disciplines. Le point fondamental est de suivre les quatre étapes de manière incessante, avec patience, certitude et discipline.

- CORPORACION CALIDAD. Premio Colombiano a la Calidad de la Gestión 2004 - 2005. Guia para participantes.

- $\quad$ CROSBY, Philip. Hablemos de calidad. McGraw Hill. 1989.

- EN: El PHVA y las Normas ISO 9000 (http://www.grupokaizen.com/sig/sig08.php). Nov. de 2005.

- EN: http://www.estrucplan.com.ar/Articulos/verarticulo.asp?IDArticulo=180. OCT 14.05. Lic. Ana María Vázquez

- JUSE. EN : http.//www.juse.or.jp/e-renme/e-r. Internet. mayo de 1999.

- PEREZ URIBE, Rafael I. La Calidad eje de la Empresa Japonesa. U. Externado de Colombia. Santafe de Bogotá, 1990.

- PEREZ URIBE, Rafael. Círculos de Calidad - Una herramienta en Calidad Total -.Alas Aeroimpresores. Abril de 1992. Santafé de Bogotá. 
- Seminario LA GERENCIA JAPONESA EN COLOMBIA Y LATINOAMERICA. Corporación de Amistad Colombo Japonesa. Memorias. Talleres de publicaciones del Sena. Primera edición. Abril de 1990. Bogotá. Colombia.

- SIERRA CORREA, Martha Lucia. Experiencia en Algunas Empresas de Santafé de Bogotá sobre Calidad Total: Estrategias y Recomendaciones para Iniciar un Proceso. Trabajo de grado presentado para optar el titulo de Administrador de Empresas. Escuela de Administración de Negocios. Director: Rafael I. Pérez Uribe. 1992.

\section{Notes et références}

1 Walther Andrew Shewhart est né en New Canton, Illinois, USA le 18 mars de 1891. Il est décédé à Troy Hills, New Jersey, USA le 11 mars 1967.

2 Une des prémisses de Deming est que lorsqu'une organisation et ses employés choisissent la qualité comme objectif, dans le temps la qualité va s'améliorer et les coûts diminuer. La qualité étant définie de la façon suivante : Qualité = Résultat Global du Travail / Coût du Travail

3 William Edwards Deming est né dans la ville de Sioux, Iowa le 14 octobre 1900, fils de William Albert Deming et Plume Irene Edwards., il est décédé le 20 décembre 1993. Sa contribution exceptionnelle à la révolution économique japonaise après la deuxième guerre mondiale fut soulignée par l'instauration en 1950 d'un prix Deming dans ce pays. Ce n'est qu'à partir de 1980 que ses théories furent finalement acceptées par les industriels américains.

4 Dans: http://www.estrucplan.com.ar/Articulos/verarticulo.asp?IDArticulo=180. OCT 14.05. Lic. Ana María Vázquez

5 Masaaki Imai est né à Tokyo en 1930. En 1955, il a reçu sa licence à Université de Tokyo, avec une spécialisation en Relations Internationales. En 1986, il a établi l'institut Kaizen pour aider les compagnies occidentales à introduire les concepts, systèmes et outils du Kaizen. La même année, il a publié son livre vedette :, «Kaizen : La clé de la compétitivité Japonaise». Depuis ce livre a été traduit en 14 langues. En mai 1997 il publie chez McGraw-Hill "Gemba Kaizen".

6 Dans: Le PDCA et les Normes ISO 9000 (http://www.grupokaizenccccc.com/sig/sig08.php). Nov. de 2005.

7 Feigenbaum est un des gourous mondiaux de la Qualité. En 1951, il a créé le concept de «Total Quality Control» et il a mis une emphase particulière sur le fait que la qualité requiert un effort total de la haute direction et ne repose pas seulement sur un département d'inspection. Il est actuellement président et Directeur général du «General Systems Company», une firme d'ingénieurs conseil qui est un des leaders mondial dans la conception et la mise en place de systèmes administratifs d'opérations et de contrôle de la qualité. Elle compte parmi ses clients plusieurs des plus grandes compagnies manufacturières et de service à travers le monde.

8 Japanese Union of Scientist Engineer fondée en 1946 sous la direction de Kaoru Ishikawa.

$9 \quad$ IBID

10 Le Professeur Kaoru Ishikawa est né au Japon en 1915 et il est décédé en 1989. Précurseur des concepts de qualité totale au Japon, il fut le premier auteur à tenter de spécifier les différences entre les styles d'administration japonais et occidentaux.

11 IBID 9

12 RAMIREZ, Gilberto : http://www.rinconaguilar.com/articulos/NegGramirez300104.htm 